\title{
Selective medium that distinguishes Haemophilus influenzae from Haemophilus parainfluenzae in clinical specimens: its value in investigating respiratory sepsis
}

\author{
D E ROBERTS, ELIZABETH HIGGS, P J COLE \\ From the Host Defence Unit, Department of Thoracic Medicine, Cardiothoracic Institute, Brompton Hospital, \\ London
}

SUMMARY A medium is described, which is selective for the haemophilus genus and also distinguishes between the species Haemophilus influenzae and Haemophilus parainfluenzae isolated in primary culture from clinical material.

Haemophilus influenzae can be distinguished from Haemophilus parainfluenzae by its growth requirements. $H$ influenzae requires both "X" factor (haemin) and " $V$ " factor (coenzyme). $H$ parainfluenzae only requires the "V" factor. Such tests using growth factor requirements can be carried out with impregnated discs ${ }^{1}$ available from Oxoid Ltd, Basingstoke. Most nutrient agar, however, contains small amounts of " $\mathrm{X}$ " factor, which enables $\boldsymbol{H}$ influenzae to grow around the disc containing " $V$ " factor alone, thereby making it seem to be independent of the " $X$ " factor and leading to its misclassification as $H$ parainfluenzae. ${ }^{2}$ One way to overcome this problem is to use a less rich medium, but then more fastidious strains fail to grow.

\section{Material and methods}

Blood agar base No 2 (Oxoid) $(40 \mathrm{~g}), 10 \mathrm{~g}$ sucrose, and $25 \mathrm{mg}$ phenol red and 1 litre distilled water were heated for 15 minutes at $121^{\circ} \mathrm{C}$. After cooling $100 \mathrm{mg}$ bacitracin (to which haemophilus is resistant) and $3 \mathrm{mg}$ haemin were added as sterile solutions and the resulting medium allowed to set in Petri dishes.

Clinical specimens were plated out in the conventional manner on surface dried medium and a " $V$ " factor disc placed on the surface. After overnight incubation at $37^{\circ} \mathrm{C}$ haemophilus colonies satellited around the " $\mathrm{V}$ " factor disc. $H$ parainfluenzae fermented the sucrose and grew as bright yellow colonies; $\boldsymbol{H}$ influenzae grew as white colonies (no indicator change).

Whenever a selective method, ${ }^{3}$ which uses anaerobic conditions to isolate haemophilus in primary cul-

Accepted for publication 8 July 1986 ture from patients with chronic respiratory sepsis complicated by carriage of pseudomonas, is required, the method described here to distinguish $H$ influenzae from $H$ parainfluenzae should be used at the subculture stage: the sucrose fermentation reaction and indicator change does not occur under anaerobic conditions, and any carbon dioxide present such as when an Oxoid gas pack is used to achieve anaerobic conditions) causes non-specific indicator colour change.

\section{SPUTUM SPECIMENS}

One hundred and fifty isolates of Haemophilus (from 107 specimens of sputum expectorated by 74 patients with chronic purulent sputum production, most with confirmed bronchiectasis, were identified by the selective method described above.

\section{Results}

The table shows the results of testing a variety of strains of haemophilus isolated in our laboratory, using a selective medium ${ }^{3}$ that incorporated no growth factors. The reference strain (NCTC 7857) and two clinical isolates (W and 253) of $H$ parainfluenzae fermented sucrose and were easily distinguishable from the reference strain (NCTC 8143) and three clinical isolates $(\mathrm{C}, \mathrm{A}, \mathrm{N})$ of $\boldsymbol{H}$ influenzae - that is, there was good correlation between sucrose fermentation, and growth factor requirements.

Using this technique 107 sputum specimens (produced by 74 patients with chronic bronchial sepsis) yielded 40 isolates of $H$ influenzae and 60 isolates of $H$ parainfluenzae, both strains being found in eight patients. 
Table Fermentation of sucrose by Haemophilus species grown on growth factor free selective medium

\begin{tabular}{|c|c|c|c|c|}
\hline \multirow[b]{2}{*}{ Strain } & \multicolumn{3}{|c|}{ Growth around disc } & \multirow[b]{2}{*}{ Sucrose fermentation } \\
\hline & “X” factor & " $V$ " factor & “X+V" factor & \\
\hline NCTC 8143 ( $H$ influenzae) & - & - & + & - \\
\hline $\mathrm{C}$ & - & - & + & - \\
\hline A & - & - & + & - \\
\hline $\mathbf{N}$ & - & - & + & - \\
\hline NCTC 7857 (H parainfluenzae) & - & + & + & + \\
\hline $\mathbf{W}$ & - & + & + & + \\
\hline 253 & - & + & + & + \\
\hline
\end{tabular}

- = no growth; + = growth.

\section{Discussion}

The clinical importance of distinguishing $H$ influenzae from $H$ parainfluenzae lies in the widely held interpretation of $H$ parainfluenzae as non-pathogenic. ${ }^{4}$ According to this, misclassification of $\boldsymbol{H}$ influenzae as $H$ parainfluenzae would result in treatment to eradicate a candidate pathogen being withheld.

We advise against over-ready acceptance of this interpretation of $\boldsymbol{H}$ parainfluenzae as non-pathogenic for four reasons: firstly, $H$ parainfluenzae has recently been reported as a pathogen in respiratory infections; ${ }^{5}$ secondly, $H$ parainfluenzae is inhibitory for human ciliary function in vitro; ${ }^{6}$ thirdly, the sputum specimens in our study yielded a surprisingly high proportion of isolates (and a study in progress documenting its presence in the bronchial tree by doublelumen bronchial brushing is yielding a similar incidence of isolates); fourthly, recent work $^{7}$ has shown that the progressive bronchial damage associated with chronic bronchial sepsis may be due to a "vicious circle" of tissue damaging inflammatory host response to a persistent microbial flora. This microbial load is largely non-invasive, avirulent, and well contained in the lung but cannot be eliminated by natural defences. It colonises an ecological niche opened by initial lung damage rather than actively invading previously normal respiratory tract in the manner of "classical" acute infection, such as pneumonia. According to this hypothesis, ${ }^{7}$ any microorganism capable of inciting an inflammatory response in the host's respiratory tract will lead to damage from its presence alone. Conventional but inadequate antimicrobial treatment may even provoke abnormal forms of organism, ${ }^{8}$ which, while evading host defences, may still evoke a tissue damaging inflammatory response. Our results showing $H$ parainfluenzae alone to be associated with chronic bronchial sepsis in the case of $\mathbf{4 0}$ patients would support the case for it being a pathogen-at least under some circumstances.
For those subscribing to the "vicious circle" hypothesis, the selective medium described here may be useful in identifying the relative roles of these micro-organisms in those processes which progressively damage the lung. For others, it may be a simple, inexpensive method of studying the carriage of such micro-organisms in health and disease.

EH is supported by Bencard. This work was financially supported by the Wellcome Trust.

\section{References}

1 Everall PH. A plate method for demonstrating the growth factor requirements of the genus Haemophilus. Journal of Medical and Laboratory Technology 1953;11:181-4.

2 Turk DC, May JR. Haemophilus influenzae: its clinical importance. London: The English Universities Press Ltd, 1967:11.

3 Roberts DE, Cole PJ. Use of selective media in bacteriological investigation of patients with chronic suppurative respiratory infection. Lancet 1980;i:796-7.

4 Smith CB, Golden CA, Kammer RE, Renzetti AD. Haemophilus influenzae and Haemophilus parainfluenzae in chronic obstructive pulmonary disease. Lancet 1976;i:1253-5.

5 Rhind GB, Gould GA, Ahmed F, Croughan MJ, Calder MA. Haemophilus parainfluenzae and Haemophilus influenzae respiratory infections: comparison of clinical features. $\mathrm{Br} \mathrm{Med}$ J 1985;291:707-8.

6 Wilson R, Pitt T, Rutman A, Roberts D, Cole PJ. Haemophilus influenzae and $H$ parainfluenzae slow and disorganise the beating of human cilia in vitro. Clin Sci 1986;70 (suppl 13):26P.

7 Cole PJ. A new look at the pathogenesis and management of persistent bronchial sepsis: a "vicious circle" hypothesis and its logical therapeutic connotations. In: Davies RJ, ed. Strategies for the management of chronic bronchial sepsis. Oxford: The Medicine Publishing Foundation, 1984:1-20.

8 Roberts DE, Higgs E, Rutman A, Cole PJ. Isolation of spheroplastic forms of Haemophilus influenzae from sputum in conventionally treated chronic bronchial sepsis using selective medium supplemented with $\mathrm{N}$-acetyl-D-glucosamine: possible reservoir for re-emergence of infection. $\mathrm{Br} \mathrm{Med} J$ 1984;289:1409-12.

Requests for reprints to: Dr P J Cole, Host Defence Unit, Department of Thoracic Medicine, Cardiothoracic Institute, Brompton Hospital, Fulham Road, London SW3 6HP. 